\title{
FIBRIN-GENIPIN ADHESIVE HYDROGEL FOR ANNULUS FIBROSUS REPAIR: PERFORMANCE EVALUATION WITH LARGE ANIMAL ORGAN CULTURE, IN SITU BIOMECHANICS, AND IN VIVO DEGRADATION TESTS
}

M. Likhitpanichkul ${ }^{1,2}$, M. Dreischarf ${ }^{3}$, S. Illien-Junger ${ }^{1,2}$, B. A. Walter ${ }^{1}$, T. Nukaga ${ }^{2,4}$, R. G Long ${ }^{1,2}$, D. Sakai ${ }^{2,4}$, A. C. Hecht ${ }^{1}$ and J. C. Iatridis ${ }^{1,2, *}$

\author{
${ }^{1}$ Leni and Peter W. May Department of Orthopaedics, Icahn School of Medicine at Mount Sinai, New York, NY, USA \\ ${ }^{2}$ Collaborative Research Partner Annulus Fibrosus Rupture Program of AO Foundation, Davos, Switzerland \\ ${ }^{3}$ Julius Wolff Institute, Charité-Universitätsmedizin Berlin, Berlin, Germany \\ ${ }^{4}$ Department of Orthopaedic Surgery, Tokai University School of Medicine, Kanagawa, Japan
}

\begin{abstract}
Annulus fibrosus (AF) defects from annular tears, herniation, and discectomy procedures are associated with painful conditions and accelerated intervertebral disc (IVD) degeneration. Currently, no effective treatments exist to repair AF damage, restore IVD biomechanics and promote tissue regeneration. An injectable fibringenipin adhesive hydrogel (Fib-Gen) was evaluated for its performance repairing large $\mathrm{AF}$ defects in a bovine caudal IVD model using ex vivo organ culture and biomechanical testing of motion segments, and for its in vivo longevity and biocompatibility in a rat model by subcutaneous implantation. Fib-Gen sealed AF defects, prevented IVD height loss, and remained well-integrated with native AF tissue following approximately 14,000 cycles of compression in 6-day organ culture experiments. Fib-Gen repair also retained high viability of native AF cells near the repair site, reduced nitric oxide released to the media, and showed evidence of AF cell migration into the gel. Biomechanically, Fib-Gen fully restored compressive stiffness to intact levels validating organ culture findings. However, only partial restoration of tensile and torsional stiffness was obtained, suggesting opportunities to enhance this formulation. Subcutaneous implantation results, when compared with the literature, suggested Fib-Gen exhibited similar biocompatibility behaviour to fibrin alone but degraded much more slowly. We conclude that injectable Fib-Gen successfully sealed large AF defects, promoted functional restoration with improved motion segment biomechanics, and served as a biocompatible adhesive biomaterial that had greatly enhanced in vivo longevity compared to fibrin. Fib-Gen offers promise for AF repairs that may prevent painful conditions and accelerated degeneration of the IVD, and warrants further material development and evaluation.
\end{abstract}

Keywords: Intervertebral disc, annulus fibrosus repair, injectable hydrogel, fibrin-genipin, adhesive biomaterial, organ culture, motion segment testing, in vivo degradation, herniation, discectomy.

Address for correspondence:

James C. Iatridis, $\mathrm{PhD}$

Leni and Peter W. May Department of Orthopaedics

Icahn School of Medicine at Mount Sinai

One Gustave L. Levy Place, Box 1188

New York, NY 10029, USA

Telephone Number: 1-212-241-1517

FAX Number: 1-212-876-3168

E-mail: James.iatridis@mssm.edu
Lower-back pain is a major health concern that causes an enormous economic burden to society and is often related to intervertebral disc (IVD) disorders, particularly IVD degeneration and herniation (Jacobs et al., 2011; Katz, 2006). Early stages of IVD disorders involve structural damage of the annulus fibrosus (AF) that initiates as internal tears and fissures, which can propagate through the outer AF layers forming radial tears most frequently located in the postero-lateral region (Osti et al., 1992; Vernon-Roberts et al., 1997). Such AF defects can lead to lower-back pain due to IVD herniation and nerve root compression (Adams and Dolan, 2012; Guterl et al., 2013; Li et al., 2014) and as a result of neurovascular ingrowth and nerve sensitisation due to oxidative stress and inflammatory conditions (Freemont et al., 1997; Purmessur et al., 2008). There is a substantial unmet clinical need to develop strategies to repair small and large $\mathrm{AF}$ defects in order to prevent and treat painful conditions to the IVD.

Lumbar discectomy is the most commonly performed spinal surgery (Deyo and Weinstein, 2001) and is used as a treatment for painful IVD protrusion and herniation that cause radicular pain. Discectomy involves removal of herniated NP tissue via the AF defect or a surgical incision to the AF that is typically left un-repaired. Chronic lowerback pain symptoms following surgical discectomy may be primarily associated with un-repaired annular defects (DePalma et al., 2012). AF injuries as small as needle puncture from discography procedures used to diagnose painful IVD are not benign and can accelerate IVD degenerative process (Carragee et al., 2009; Iatridis and Hecht, 2012; Iatridis et al., 2013; Michalek et al., 2010). Currently there is still no effective treatment method to successfully repair annular defects. When painful degenerative processes are allowed to accelerate, complete IVD removal with a spinal fusion procedure becomes the 'gold standard' (Chan and Gantenbein-Ritter, 2012a). A minimally invasive annular repair strategy that is capable of preventing or slowing the accelerated IVD degeneration due to AF injury remains a high research priority.

Several AF repair methods have been developed with limited success because of the challenge that successful repair needs to be able to immediately withstand complex spinal load and restore and maintain IVD function to prevent re-herniation. Suture repair of AF incision alone did not significantly improve the healing strength of lumbar IVD following discectomy in a sheep model (Ahlgren et al., 2000). AF closure devices, such as barb-ringed polyethylene plugs, were found to expel or 
(a)

Bovine IVD Samples

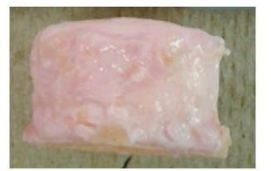

Intact

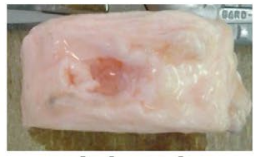

Injured

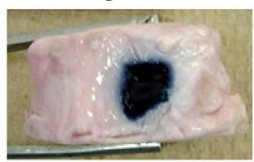

Repaired (b) Bioreactor

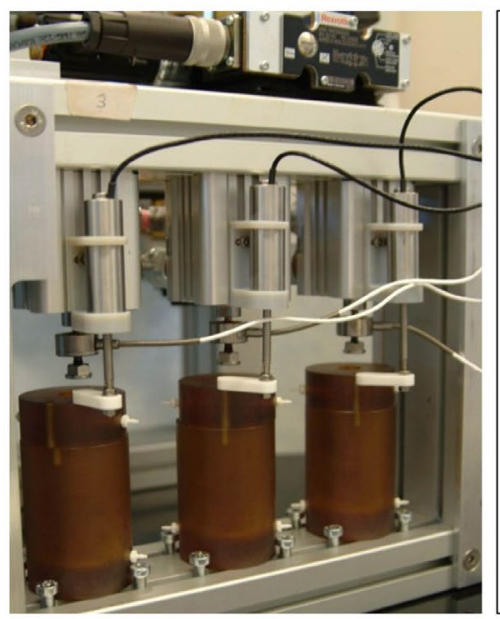

(c)

Daily Loading Regime

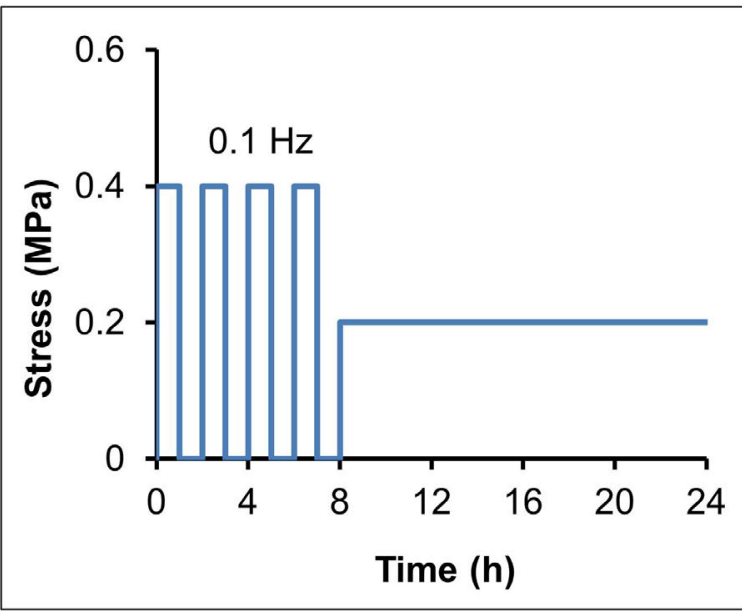

Fig. 1. Study design for Fib-Gen repair using IVD organ culture model. (a) Bovine caudal IVD samples that retained IVD endplates were evaluated as Intact, Injured (with simulated 4.5 x $4.5 \mathrm{~mm}$ box-cut defect) and Repaired (injured with Fib-Gen injection for repair). (b) Hydraulically controlled dynamic compression bioreactor with culture chambers. (c) Simulated daily activity load consisted of $8 \mathrm{~h}$ of cyclic compression and $16 \mathrm{~h}$ of static resting load.

damage the endplates, with signs of IVD destruction and displacement after 6 weeks in a goat model (Bron et al., 2010). Application of biomaterials, such as hydrogels, bioglass, collagen silks and degradable polymers (Iatridis et al., 2013), to promote tissue regeneration utilising a tissue engineering approach for AF repair offers some promise. However, most developed biomaterials are soft hydrogels with mechanical properties that are orders of magnitude lower than native AF tissues, and are not able immediately to withstand the high loading demand of the IVD. Therefore such biomaterials may not contribute to load support or withstand high mechanical load in the AF until tissue regeneration and integration with surrounding AF tissue occurs.

We developed the following design requirements for a biomaterial to repair AF defects: 1) strongly adhesive to native AF; 2) biomechanically tuned to match $\mathrm{AF}$ mechanical properties; 3) biocompatible; 4) able to withstand repetitive loading until resorption; 5) injectable and fast setting for easy delivery to injury site at time of surgery. Fibrin is widely used as a sealant for general tissue repair and was utilised to repair de-nucleated IVDs in a minipig model (Buser et al., 2011), but the gel's mechanical properties may not be suitable for repair of load-bearing tissues. Genipin is a natural cross-linking agent derived from Gardenia fruit, has been shown to have low toxicity (Sung et al., 1999), and provides enhanced stiffness when cross-linked with fibrin (Dare et al., 2009). Our group further developed the fibrin-genipin hydrogel (Fib-Gen) for AF repair with gel composition optimised to obtain an adhesive hydrogel that possesses shear properties approaching those of native AF, and adhesive strength comparable to fibrin gel alone (Schek et al., 2011). FibGen was shown to be cytocompatible with human AF cells in $2 \mathrm{D}$ culture and could be enhanced with the addition of matrix proteins (Guterl et al., 2014). While Fib-Gen shows promise as an AF repair material, in situ and in vivo evaluations are required to better assess feasibility for the human spinal environment. The first aim of this study was to evaluate the performance of Fib-Gen gel in repairing large AF defects using a large animal organ culture model which allowed for evaluation of both the functional performance and the cytocompatibility of the gel under well-controlled loading and culture conditions. We hypothesised that Fib-Gen, with its enhanced mechanical properties, can withstand repetitive loading under culture conditions, contribute to improved IVD functional performance, and is cytocompatible with IVD cells. The second aim was to perform a rigorous biomechanical assessment of the Fib-Gen repair in situ, using bovine motion segment testing under both axial and torsional loading, with the hypothesis that AF repair with Fib-Gen can improve the biomechanics of injured IVDs. Finally, the third aim was to assess the in vivo degradation rate and the biocompatibility of Fib-Gen by the subcutaneous implantation of Fib-Gen in rats with the hypothesis that Fib-Gen has extended longevity and is biocompatible in vivo.

\section{Materials and Methods}

\section{Organ culture}

A bovine caudal IVD organ culture model was used to assess the functional and biological performance of FibGen for AF repair of injured IVDs. Bovine caudal IVDs were used because of their large size and similarities with human lumbar IVDs (Demers et al., 2004; Oshima et al., 1993). In addition, bovine and other large animal IVDs are readily available and have been thoroughly characterised biomechanically and in organ culture studies (Chan et al., 2011; Gantenbein et al., 2006; Haglund et al., 2011; 
Illien-Junger et al., 2012; Junger et al., 2009; Korecki et al., 2008a; Korecki et al., 2008b; Lee et al., 2006; Malonzo et al., 2013; Michalek and Iatridis, 2012; Ohshima et al., 1995; Paul et al., 2012; Walter et al., 2011).

\section{Experimental set-up}

Twelve IVDs were harvested from 3 caudal levels (cc12 to cc3-4) of 4 skeletally mature bovine tails obtained from a local abattoir, and assigned to Intact (control), Injured, and Repaired groups ( $n=4$, Fig. 1a). IVDs with retained endplates were harvested by cutting the vertebrae proximal and distal to vertebral endplates with a histological band saw (Exakt 310, Exakt, Norderstedt, Germany). The endplates were flushed with water using an orthopaedic irrigation debridement system (Inter Pulse ${ }^{\circledR}$, Stryker, Kalamazoo, MI, USA) to remove blood clots and bone debris (Chan and Gantenbein-Ritter, 2012b; Gantenbein et al., 2006). IVDs were then rinsed in ethanol, phosphate buffered saline (PBS) containing $3 \%$ penicillin/ streptomycin and $1.5 \%$ Fungizone (Invitrogen, Carlsbad, CA, USA) and PBS. To account for potential differences in IVD level-dependent cell metabolism rates (Wiseman et al., 2005) IVDs from each tail were randomly assigned into the three groups. For Injured and Repaired IVDs, posteriorlateral box-cut defects of $4.5 \times 4.5 \mathrm{~mm}$ that penetrated to the centre of the disc were created using a \#11 scalpel blade. The box-cut defect approximately simulated the size and sharp features of a large AF defect that would be found following a micro-discectomy procedure, but in this case most of the NP material was retained. In rabbit and other species, even relatively small needle puncture injuries are known to induce IVD degeneration (Iatridis et al., 2013; Masuda et al., 2005; Sobajima et al., 2005), so this box-cut defect was considered to be a 'critical-sized' defect and expected to progress to degeneration in an in vivo context.

Fib-Gen gel formulation that contained final concentrations of $140 \mathrm{mg} / \mathrm{mL}$ fibrinogen, $28 \mathrm{U} / \mathrm{mL}$ thrombin and $6 \mathrm{mg} / \mathrm{mL}$ genipin was prepared and filled into the defects in the Repaired group using a 4:1 dualbarrel syringe with a mixer tip (Sulzer mixpac, Winterthur, Switzerland). This gel formulation was previously optimised to match AF shear material properties and has been shown to be biocompatible with cultured human AF cells (Guterl et al., 2014; Schek et al., 2011). Preparation of Fib-Gen gel involved; (1) fibrinogen isolated from bovine plasma (Sigma-Aldrich, St. Louis, MO, USA) was dissolved in PBS at a concentration of $176 \mathrm{mg} / \mathrm{mL}$, and (2) thrombin isolated from bovine plasma (Sigma Aldrich) was dissolved in PBS at a concentration of $100 \mathrm{U} / \mathrm{mL}$ and genipin (Wako, Richmond, VA, USA) was dissolved in dimethyl sulphoxide (Fisher Scientific, Hampton, NH, USA) at a concentration of $400 \mathrm{mg} / \mathrm{mL}$. A mixture of $141 \mathrm{U} / \mathrm{mL}$ thrombin and $28 \mathrm{mg} / \mathrm{mL}$ genipin within $1 / 4$ volume of (1) was prepared. Mixtures (1) and (2) were then pipetted into large and small compartments of the 4:1 dual-barrel syringe respectively and kept in a $37{ }^{\circ} \mathrm{C}$ water bath until ready for use.

IVDs were loaded into a custom-designed hydraulicallycontrolled bioreactor system (Walter et al., 2014) (Fig. 1b) and cultured for $6 \mathrm{~d}$ under a simulated daily load consisting of $8 \mathrm{~h}$ of dynamic compression up to $0.4 \mathrm{MPa}$ at $0.1 \mathrm{~Hz}$ frequency, followed by $16 \mathrm{~h}$ of resting static load at $0.2 \mathrm{MPa}$, resulting in approximately 14,000 cycles of compression in total after the 6-day culture period (Fig. 1c). All cultures were performed at $37{ }^{\circ} \mathrm{C}$ with hypoxia $\left(5 \% \mathrm{CO}_{2}, 5 \% \mathrm{O}_{2}\right)$ using standard culture media consisting of high glucose DMEM, $10 \%$ FBS (Invitrogen, Carlsbad, CA, USA), $1 \%$ Pen/Strep, $50 \mu \mathrm{g} / \mathrm{mL}$ ascorbic acid (FisherScientific, Waltham, MA, USA) and 1:500 Primocin (Invivogen, San Diego, CA, USA). The value of $5 \% \mathrm{O}_{2}$ was used because it has been reported that the oxygen tension is $\sim 4 \%$ in capillaries (Intaglietta et al., 1996), and $\sim 5-7 \%$ in the IVD near the periphery of the outer AF and the endplates (Holm et al., 1981). The medium was changed at day 4 .

\section{Histology}

Following culture, all IVDs were cut into two halves for histology and cell viability analyses. Injured and Repaired IVDs were cut through the center of the defect and the Fib-Gen repair site. Cross sections of Injured and Repaired IVDs were visualised for a macroscopic assessment of the defect and structural repair with Fib-Gen. One half of the IVDs were then fixed in Z-fix fixative (Fisher-Scientific) and embedded in methacrylate resin. 5 - $\mu \mathrm{m}$ thin histological sections were cut, and stained with X-FAST staining (Leung et al., 2009). The structural repair with Fib-Gen was assessed microscopically with a focus on the Fib-Gen/ AF tissue interface.

\section{IVD height measurement}

The key assessment of the functional performance of Fib-Gen repair in culture was a comparison of the total IVD height loss among the three groups. IVD height measurements were taken before and after culture, from endplate to endplate at 3 different random locations using digital callipers (Mitutoyo 500-196, Aurora, IL, USA, accuracy $\pm 0.025 \mathrm{~mm}) . \%$ IVD height loss was calculated from changes in the IVD height before and after $6 \mathrm{~d}$ in culture with approximately 14,000 cycles of compression. IVD height was measured immediately after the culture at the end of the cyclic loading period so that the IVD height loss included the change from the last loading cycle and any change that might have occurred over the whole culture period. ANOVA with Tukey's post-hoc statistical analysis was performed to compare among the three IVD specimen groups, with $p<0.05$ considered significant.

\section{Cell viability}

Cell viability was assessed using 3-(4,5-dimethylthiazol2-yl)-2,5-diphenyltetrazolium bromide (MTT, SigmaAldrich; live, blue/black precipitate) and 4',6-diamidino2-phenylindole (DAPI, Roche Diagnostics, Indianapolis, IN, USA; nuclei stain, blue fluorescence) as previously described (Korecki et al., 2007). Following culture, tissue sections were dissected through the repair site and placed into DMEM containing $1 \mathrm{mg} / \mathrm{mL}$ MTT for $3 \mathrm{~h}$. Tissue was washed, flash frozen and stored at $-80^{\circ} \mathrm{C}$ until sectioning on a cryotome. Three x $10 \mu \mathrm{m}$ thick sections were taken from each IVD, beginning at the tissue surface and every $100 \mu \mathrm{m}$ thereafter. Slides were fixed, counter-stained with DAPI, and cover slipped. Representative images 
(a)

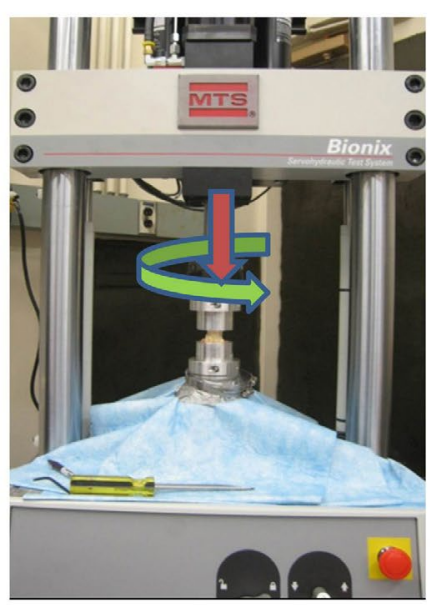

(b)

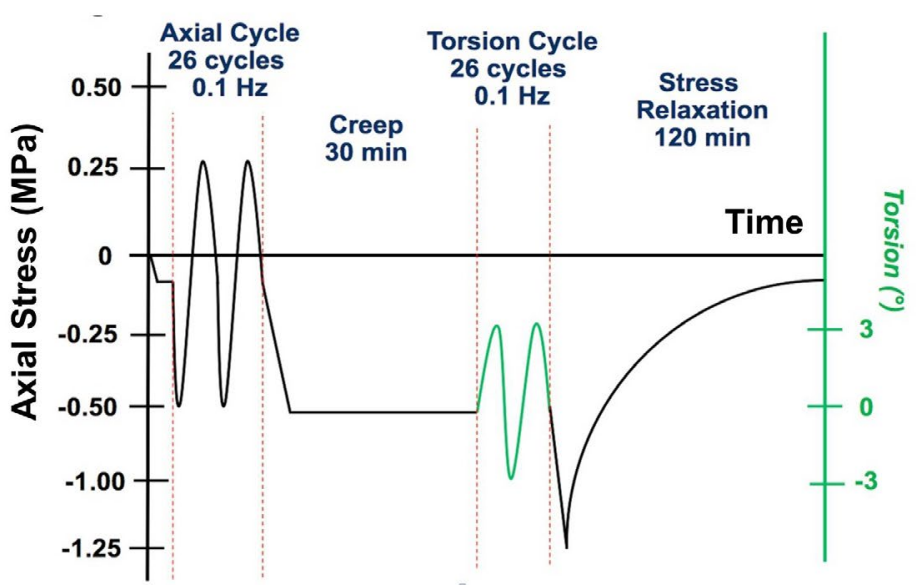

(c)
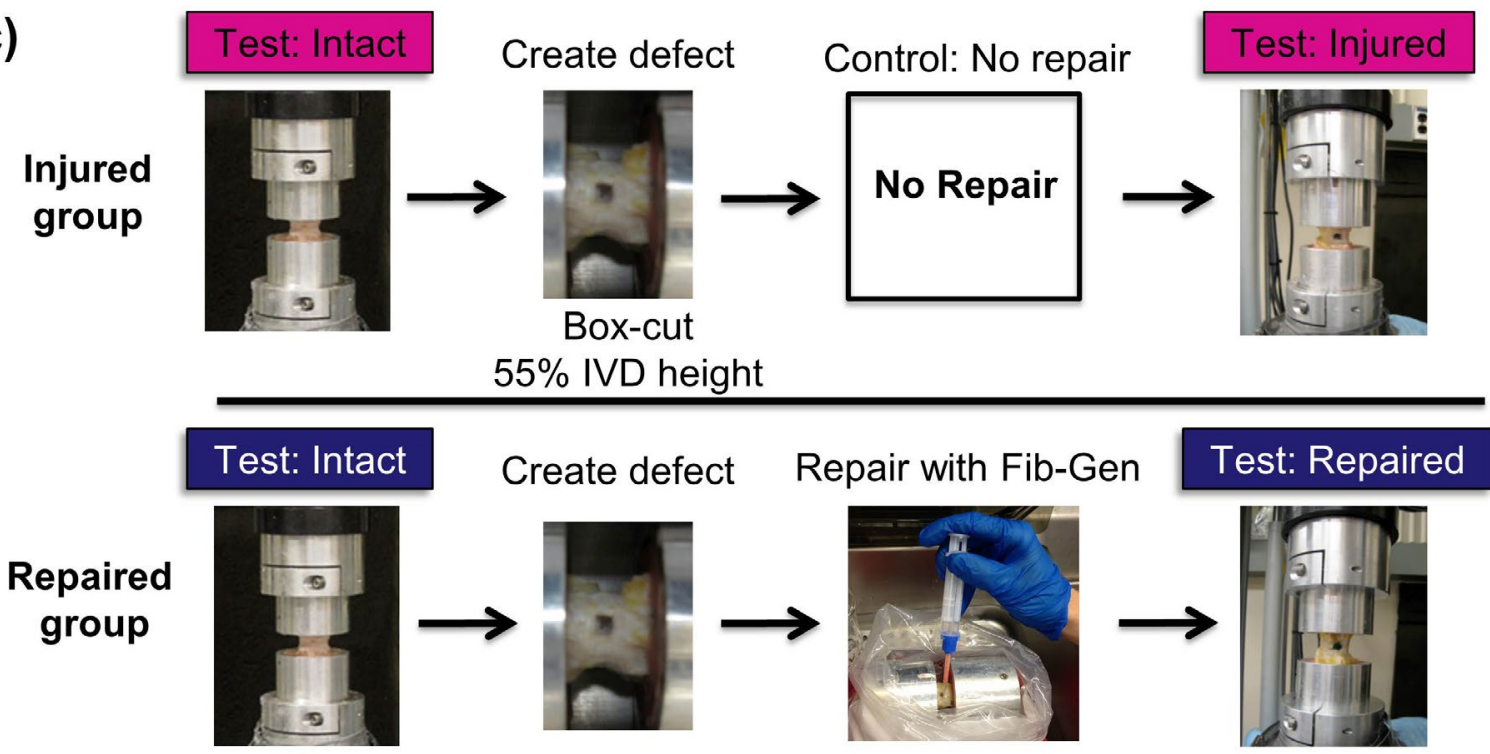

Fig. 2. Study design for Fib-Gen repair using in vitro biomechanical testing. (a) Multi-axis MTS testing frame for axial and torsional loading. (b) Loading scheme for cyclic tension-compression, cyclic torsion and stress relaxation tests. (c) Assigned groups and schematic of preparation and testing procedures for biomechanical testing of bovine caudal motion segments.

were captured at 20x magnification first under fluorescent light to capture cell nuclei stained with DAPI (Ultraviolet filter) and then under bright field light to capture vital cells stained with MTT; the images were then merged. The total number of cells (DAPI) and dual stained cells (DAPI+MTT) were counted using ImageJ (Schneider $e t$ al., 2012) and a viability percentage Live (MTT+DAPI)/ Total (DAPI) was calculated for each image.

\section{Cell migration}

To assess cell migration into the Fib-Gen gel, $10 \mu \mathrm{m}$ thick cryo-sections of the tissue Fib-Gen gel interface were stained with DAPI to visualise the cell nuclei in areas near the Fib-Gen/AF tissue interface. Cell migration into the Fib-Gen gel was further visualised on a confocal laser scanning microscope (Leica SP5 DMI, Buffalo Grove, IL, USA). A Fib-Gen gel fragment from the repair site was stained with Calcein AM and Hoechst, and a 3-D reconstruction of $175 \mu \mathrm{m}$ deep scans into the Fib-Gen gel was created.

\section{Nitric oxide (NO) release}

NO released into the media at day 4 was measured using a standard Griess reagent assay (Promega, Madison, WI, USA) according to manufacturer's instruction and normalised to the IVD weight before culture. Repeated measures ANOVA with Tukey's post-hoc statistical analysis was performed to compare among the three IVD specimen groups, with $p<0.05$ considered significant.

\section{Biomechanical tests of motion segments}

Successful AF repair should provide biomechanical restoration of the IVD motion segment. While the functional performance of the Fib-Gen repair was partially assessed with compressive mechanical loading during organ culture, a more thorough mechanical evaluation was required. We performed in vitro biomechanical testing of bovine motion segments to further test the Fib-Gen repair performance in situ under axial and torsional testing modes using 'acute' biomechanical testing regime in order to determine changes in the biomechanical parameters from 
injury and to test whether Fib-Gen can restore the changes back to the intact condition.

\section{Specimen preparation}

Twelve bone-disc-bone motion segments (cc 2-3 to cc 3-4) were harvested from skeletally mature bovine tails. IVDs were wrapped in saline-soaked gauze, and motion segments were frozen at $-20^{\circ} \mathrm{C}$ until testing. Prior to each mechanical test, specimens were thawed and rehydrated for $18 \mathrm{~h}$ at $4{ }^{\circ} \mathrm{C}$ in PBS to ensure a uniform initial hydrated condition (Johannessen et al., 2004; Malhotra et al., 2012). Motion segments were potted in polymethylmethacrylate (PMMA) following placement of wood screws into the vertebrae to improve pull-out strength. Potted specimens were rehydrated in PBS at room temperature for $1 \mathrm{~h}$ to account for possible water loss during potting, and then X-rayed to evaluate IVD orientation, anatomical alignment and assess IVD height. A custom-written Matlab code (Mathworks, Natick, MA, USA) was used to measure IVD height from the average distance between the two traced endplates that were fitted with a cubic spline function. The mean IVD diameter was determined by four measurements using callipers, assuming a circular IVD cross sectional shape.

\section{Mechanical testing protocol}

Mechanical testing was performed on an MTS Bionix Servohydraulic Test system (MTS, Eden Prairie, MN, USA; Fig. 2a) within a saline bath. The combined axial and torsional testing regime (Fig. $2 b$ ) began with 25 sinusoidal cycles of tension-compression loading from $0.5 \mathrm{MPa}$ in compression to $0.25 \mathrm{MPa}$ in tension at $0.1 \mathrm{~Hz}$. The peak compressive load of $0.5 \mathrm{MPa}$ was chosen in accordance with a physiological spinal load during unsupported standing measured in human lumbar spine (Sato et al., 1999; Wilke et al., 1999). The peak tensile load of $0.25 \mathrm{MPa}$ was sufficient for measurement of the neutral zone and tensile properties and was consistent with the level previously used (Johannessen et al., 2006). After the cyclic axial loading, a creep period of $30 \mathrm{~min}$ at $0.5 \mathrm{MPa}$ in compression was allowed and was immediately followed by 25 cycles of torsional loading of $\pm 3^{\circ}$ rotation at $0.1 \mathrm{~Hz}$. Torsion testing followed the creep period in order to equilibrate the load and place the AF fibres in circumferential tension in order to better simulate physiological conditions as has been applied previously (Showalter et al., 2012). The peak torsional angle of $3^{\circ}$ was chosen in accordance with in vivo values measured in human under maximal voluntary motion (Pearcy and Tibrewal, 1984; Steffen et al., 1997). The 25th cycle for each loading mode was selected for data analysis to ensure that a dynamic equilibrium was reached (Johannessen et al., 2006). Axial and torsional tests were both performed at $0.1 \mathrm{~Hz}$ since this frequency is within the physiological range and is consistent with prior studies (Elliott et al., 2008; Michalek and Iatridis, 2012). Finally, a stress relaxation test was performed with a ramped compressive load up to $1.25 \mathrm{MPa}$ and the final peak displacement was kept constant for $120 \mathrm{~min}$. Tensile and compressive stiffness were calculated by a linear regression of the load-displacement curve between $60 \%$ and $100 \%$ of the maximal load in the unloading part of the $25^{\text {th }}$ cycle. Axial range of motion (RoM) was calculated as the total peak-to-peak displacement. Torsional stiffness was calculated from a linear regression of the torque-rotation curve of the $25^{\text {th }}$ cycle. The torque range was directly determined from the total peak-to-peak torque. The stress relaxation behaviour was fitted with a stretched exponential model, to obtain the stress relaxation time constant $(\tau)$.

$$
F=F_{\infty}+\left(F_{0}-F_{\infty}\right) \times e^{-(t / \tau)^{\beta}}
$$

Potted motion segments were assigned to Injured $(n=6)$ and Repaired groups $(n=6)$ (Fig. 2c). Specimens in both groups were first tested to obtain biomechanical parameters under an intact condition. In both specimen groups, box-cut defects were created posterolaterally using a \#11 scalpel blade with the defect size controlled to be $55 \%$ of the IVD height of each specimen, while the defect depth was controlled to penetrate to the IVD centre. It has been shown that the defect size to IVD height ratio over $40 \%$ can induce significant alteration in biomechanical parameters (Elliott et al., 2008). The standardised defect allowed for the defect size to IVD height ratio to be kept constant, reducing the effect of specimen size variation. In the Repaired group, the defects were later repaired with Fib-Gen, as described above. Specimens in both groups were kept for $18 \mathrm{~h}$ under physiological conditions at $37^{\circ} \mathrm{C}$ in a PBS bath to allow a complete rehydration and recovery (Johannessen et al., 2004; Malhotra et al., 2012), and for Fib-Gen to fully crosslink. During this period, all specimens were treated with protease inhibitor (Roche Diagnostics, Indianapolis, IN, USA) to prevent tissue degradation, and this process was found to have negligible effect on the IVD mechanical properties based on a test-retest pilot experiment on intact motion segments. Injured and Repaired specimens were then retested using the same test protocol to allow a direct comparison of mechanical properties as compared to the corresponding intact condition. This repeated measure design was chosen to eliminate animal and level variation, and has been effectively used in prior spine biomechanical studies (Malhotra et al., 2012).

Torsional and axial biomechanical parameters were determined in bovine motion segments from the Injured and Repaired group. Restoration of motion segment biomechanics with Fib-Gen repair was reported as the ratio of each parameter calculated under the injured or repaired condition normalised to that under the intact condition of the same specimen, where a ratio of 1 represents full restoration to the intact state.

\section{Statistical analysis}

Student's paired $t$-tests were performed to compare biomechanical parameters measured under injured or repaired condition in comparison to the intact condition within the same specimen, and to compare the ratio of each biomechanical parameter (injured/repaired condition normalised to intact condition) between the Injured and Repaired group, with $p<0.05$ considered significant.

\section{Subcutaneous implantation}

Cylindrical discs of Fib-Gen $(5 \mathrm{~mm}$ diameter, $1.5 \mathrm{~mm}$ thickness) were prepared using a custom-made mould under sterile condition. Following gelation, Fib-Gen 

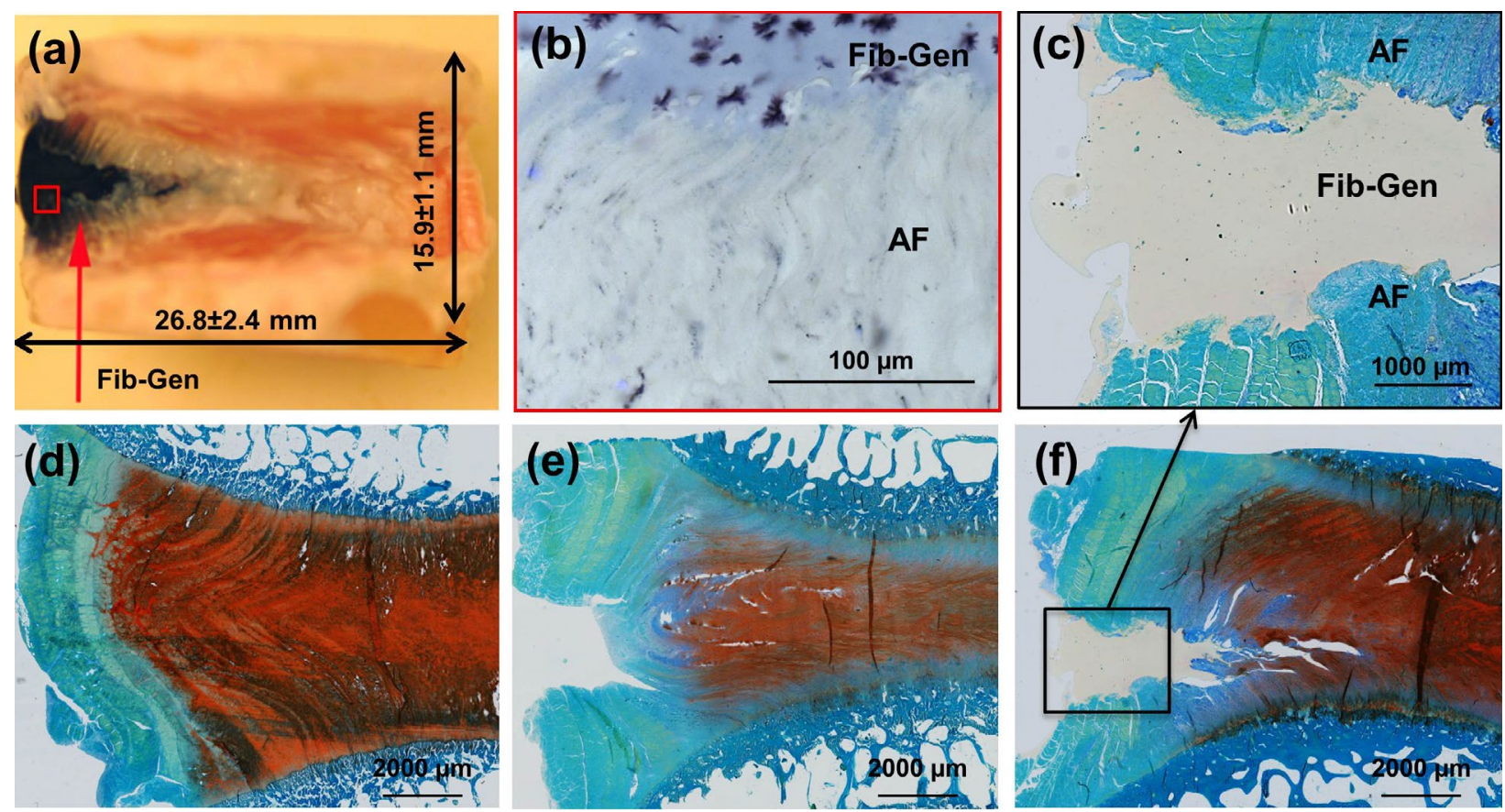

Fig. 3. Fib-Gen repair on sagittal sections taken at Day 6. (a) Macroscopic observation of repaired IVD demonstrated that injected Fib-Gen filled the IVD defect and was easy to identify because it turned blue upon crosslinking. Some leaching of the blue cross-linked material was also present in adjacent AF tissue. (b) Microscopic imaging from cryosections demonstrated good interpenetration of Fib-Gen with the collagenous AF structure. (c-f) Methacrylate sections stained with extended FAST showed that (d) Intact IVDs exhibited strong blue staining of the organised outer AF region and strong red staining of the GAG-rich inner AF and NP regions; (e) Injured IVDs exhibited a disrupted AF with loss of GAG and IVD height; and $(\mathbf{c}, \mathbf{f})$ Fib-Gen Repaired conditions demonstrated the defect remained filled with Fib-Gen following approximately 14,000 cycles of simulated physiological loading, retained IVD height, had strong red GAG staining, and confirmed strong adhesion of Fib-Gen with the native AF tissue. No evidence for remodelling at the interface was observed (or expected) at this time point in these organ culture experiments.

discs were implanted into the dorsal subcutaneous tissue of 9 to 11 week old Sprague-Dawley rats. Rats were anaesthetised with isoflurane gas and three FibGen discs were inserted into $1-\mathrm{cm}$ incision pockets at three different locations of the dorsal subcutaneous space within each rat. After 8 and 16 weeks, rats were sacrificed and implants with surrounding tissue were harvested. Photographs of remaining Fib-Gen discs were taken to measure the changes in diameter. Harvested implants were then fixed in $4 \%$ paraformaldehyde and embedded in parafin. Histological sections were cut, stained with H\&E and microscopically evaluated for the qualitiative characteristics of inflammatory response within the surrounding tissue and gel to assess the in vivo biocompatibility of Fib-Gen.

\section{Results}

\section{Organ culture}

Following approximately 14,000 cycles of compressive load under the 6-day organ culture condition, the defects created during the simulated injury remained to be sealed with Fib-Gen, as observed macroscopically (Fig. 3a) and microscopically (Fig. 3b). Microscopic assessment of the Fib-Gen/AF interface in cryo-sections demonstrated that Fib-Gen seeped into cracks and spaces between collagen

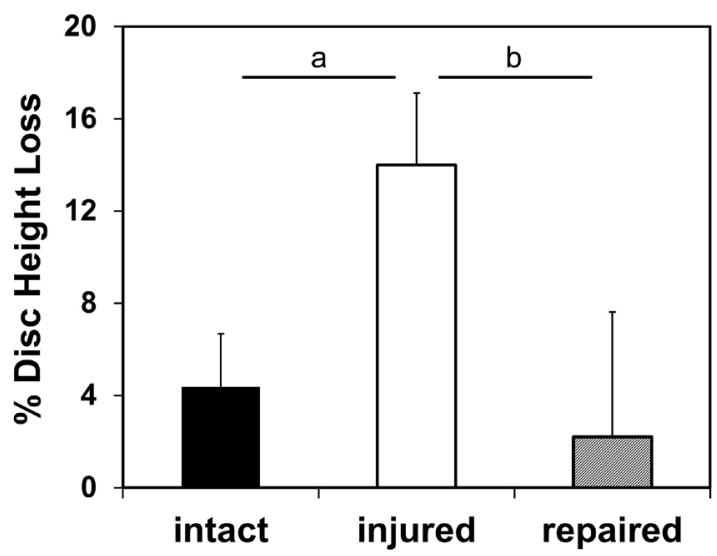

Fig. 4. Percent disc height loss for organ culture study. Intact and Repaired IVDs maintained similar values of disc height loss well below $5 \%$, while the Injured condition had significantly more disc height loss than either Intact or Repaired IVDs. $\mathrm{a}=p<0.01, \mathrm{~b}=p<0.05$.

fibres within the AF structure (Fig. 3b). Histological sections stained with X-FAST (Fig. 3c) also demonstrated that Fib-Gen successfully integrated with the AF, as no sign of cracks or gaps between the Fib-Gen gel and the AF tissue were observed, suggesting Fib-Gen effectively sealed the defect and maintained adherence with AF throughout the entire culture period. Intact IVD exhibited strong blue/ 
Fig. 5. Viability imaging and NO in media. (a) Viability imaging using cryosections stained with DAPI/MTT exhibiting live cells in the AF demarcated with black arrows (live $=$ doublestained black and blue, dead = stained for DAPI alone in blue). Migration of cells into Fib-Gen was also observed as demarcated by white arrows. (b) Confocal images further document cell migration (cyan) into the fractured interface boundary of the Fib-Gen (red colour due to autofluorescence). (c) NO levels with FibGen repair were restored to intact levels. $\mathrm{a}=p<0.01, \mathrm{~b}=p<0.05$.
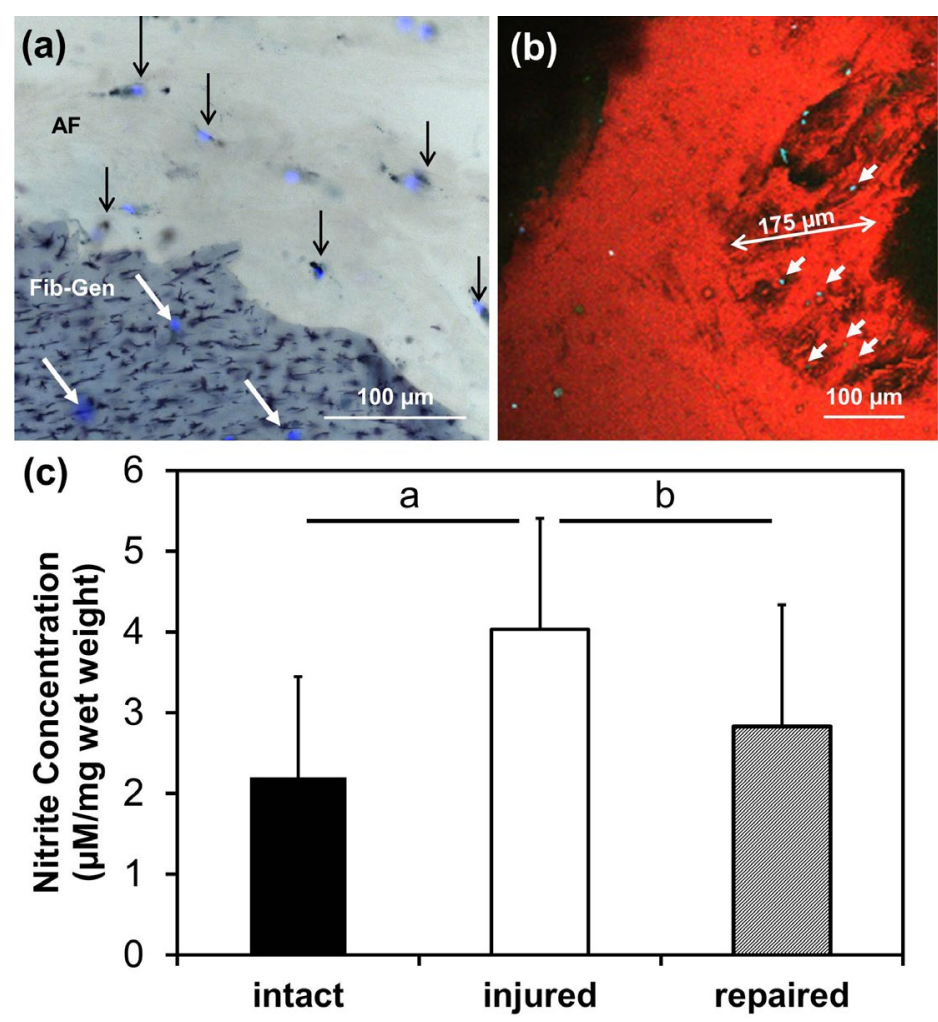

Table 1. Mean $\pm \mathrm{SD}$ results from biomechanical testing.

\begin{tabular}{|c|c|c|c|c|c|c|}
\hline \multirow[b]{2}{*}{ Measured Properties } & \multicolumn{3}{|c|}{ Injured group (un-repaired control) } & \multicolumn{3}{|c|}{ Repaired group } \\
\hline & Intact & Injured & Ratio & Intact & Repaired & Ratio \\
\hline Compressive stiffness (N/mm) & $655.0 \pm 107.3$ & $717.9 \pm 91.9^{a}$ & $1.10 \pm 0.07$ & $700.1 \pm 154.2$ & $710.3 \pm 139.4$ & $1.02 \pm 0.05$ \\
\hline Tensile stiffness $(\mathbf{N} / \mathbf{m m})$ & $359.5 \pm 66.3$ & $327.4 \pm 55.2^{b}$ & $0.91 \pm 0.02$ & $321.4 \pm 60.5$ & $307.0 \pm 60.6$ & $0.96 \pm 0.05$ \\
\hline Axial range of motion $(\mathrm{mm})$ & $2.8 \pm 0.8$ & $3.3 \pm 0.82$ & $1.16 \pm 0.12$ & $2.7 \pm 1.2$ & $2.9 \pm 1.1$ & $1.07 \pm 0.09$ \\
\hline Stress relaxation time constant & $563.6 \pm 85.7$ & $469.1 \pm 78.0$ & $0.83 \pm 0.03$ & $598.7 \pm 149.6$ & $514.6 \pm 117.8$ & $0.87 \pm 0.10$ \\
\hline Torsional stiffness $\left(\mathrm{Nm} /{ }^{\circ}\right)$ & $1.2 \pm 0.3$ & $0.9 \pm 0.2^{b}$ & $0.73 \pm 0.06$ & $1.1 \pm 0.3$ & $0.9 \pm 0.3^{b}$ & $0.79 \pm 0.05$ \\
\hline Torque range $(\mathbf{N m})$ & $2.5 \pm 0.8$ & $1.7 \pm 0.4^{b}$ & $0.72 \pm 0.07$ & $2.4 \pm 0.9$ & $1.9 \pm 0.7^{b}$ & $0.79 \pm 0.05$ \\
\hline
\end{tabular}

Bold indicates a significant difference from the Intact. $\mathrm{a}=p<0.05, \mathrm{~b}=p<0.01$.

green staining of the organised outer AF region and strong red staining of the GAG-rich inner AF and NP regions (Fig. 3d). Injured IVDs exhibited a disrupted AF with loss of GAG and IVD height (Fig. 3e). The Fib-Gen Repaired IVD showed retained IVD height, and had strong red GAG staining, suggesting that Fib-Gen repair prevented IVD structural changes from injury.

The IVD height loss compared to day 0 following approximately 14,000 cycles of compressive load was not statistically different between the Repaired (2.2 $\pm 5.4 \%)$ and Intact groups $(4.4 \pm 2.3 \%)$, but there was a highly significant decrease of $14 \pm 3.1 \%$ IVD height in the Injured group ( $p<0.01$, Fig. 4). Fib-Gen not only withstood the repetitive load but prevented IVD height loss from injury.

Cell viability was maintained in the repaired IVDs at very high levels, for both AF cells surrounding the repaired defects $(93.4 \pm 2.9 \%)$ (Fig. 5a) and NP cells (91.1 $\pm 7.2 \%)$. IVD cells were found to have migrated into the gel (Fig. $5 a, b)$. Reconstruction of confocal images of a fractured interface boundary of the Fib-Gen also showed migration of viable cells into the gel (Fig. 5b). Measured NO released into the media normalised to specimen weight at day 4 (Fig. 5c) from the Injured group (4.03 \pm 1.37$)$ significantly increased compared to the Intact group $(2.20 \pm 1.25$, $p<0.01)$. The Fib-Gen Repaired group $(2.83 \pm 1.50)$ was significantly lower than the Injured group $(p<0.05)$, but was not statistically different from the intact group.

\section{Biomechanical performance in motion segments}

Injury-induced alterations of several biomechanical parameters, and the Fib-Gen was able to restore some of those parameters (Table 1). A typical axial forcedisplacement curve from the cyclic tension-compression test of an injured specimen is shown in Fig. 6a. Results indicated significant difference between Repaired and Injured groups in the ratio of the compresive stiffness when normalised to the corresponding intact condition $(p<0.05$, Fig. 6b). It is notable that the injury counter-intuitively increased the compressive stiffness ( $p<0.05$, Fig. 6b) from the intact condition. Importantly, this compressive stiffening due to injury was eliminated with Fib-Gen repair. For tensile stiffness, there was a trend of an increase in 
(a)

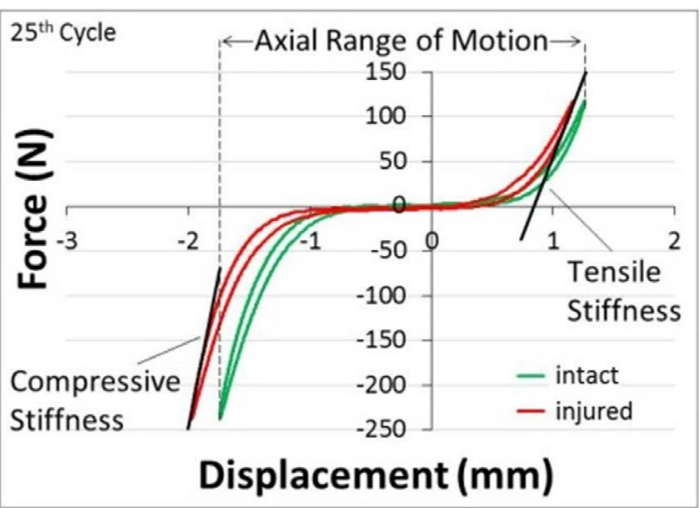

(b)

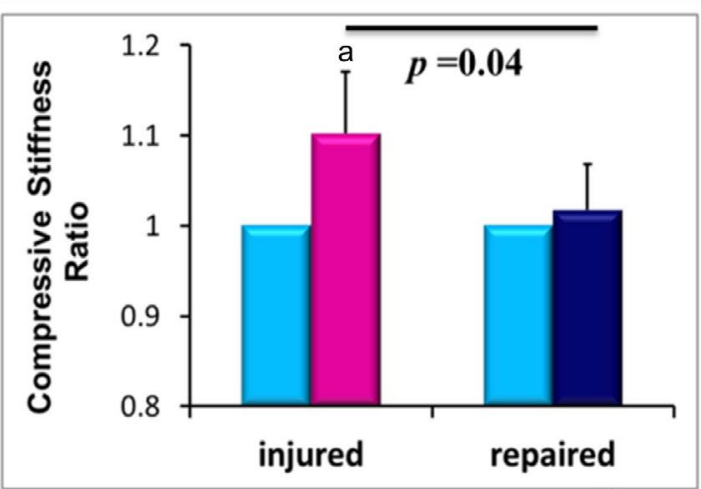

(c)

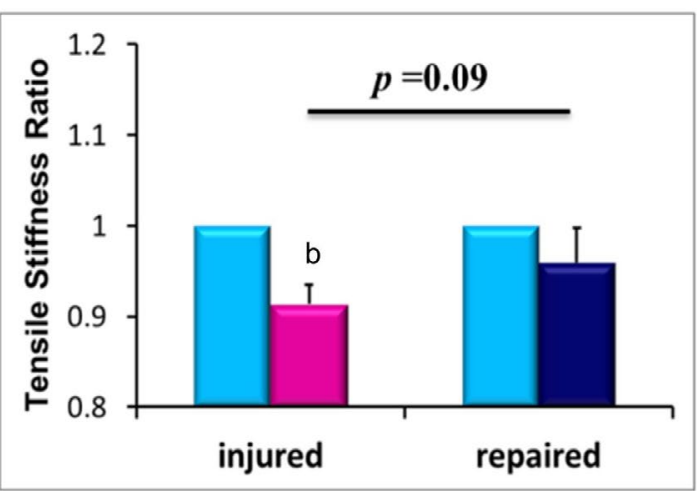

(d)

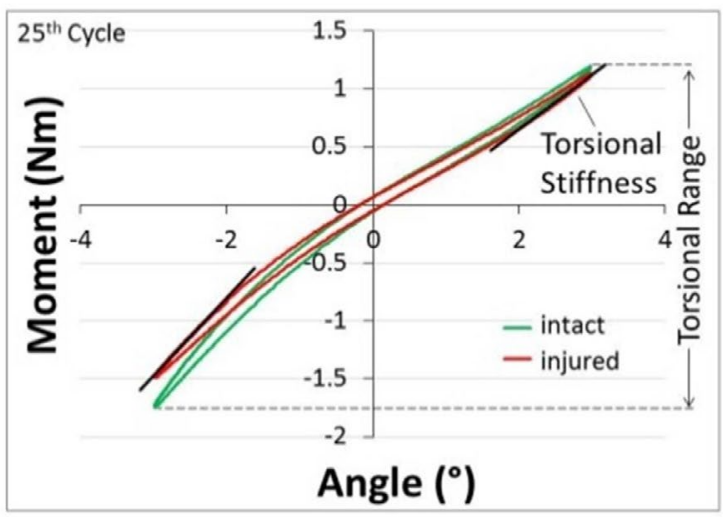

(e)

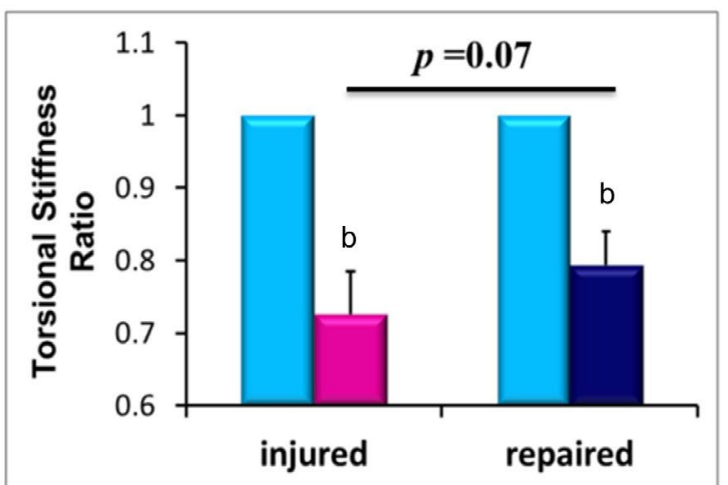

(f)

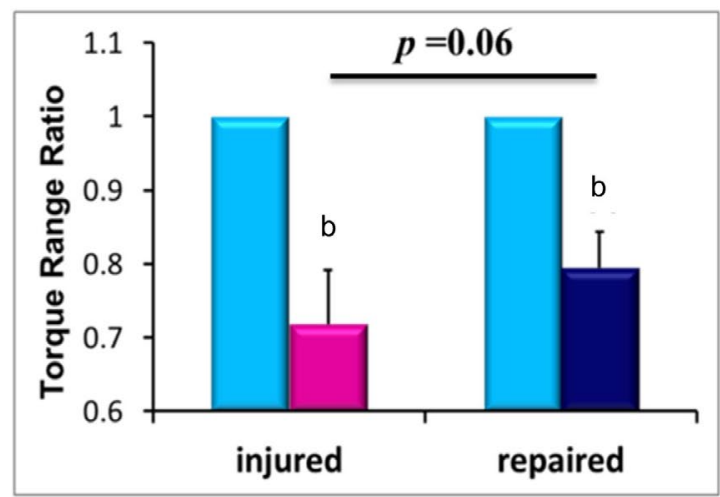

Fig. 6. Biomechanical test results. (a) Typical axial force-displacement curve from tension-compression test of an injured specimen along with comparisons between groups for (b) Compressive stiffness and (c) Tensile stiffness ratios (i.e., compared to the intact IVD for each test). Axial biomechanical parameters were partially or completely restored to Intact levels for the Fib-Gen repair group. (d) A typical torque-rotation curve from torsional test of an injured specimen along with comparisons between groups for (e) Torsional stiffness and (f) Torque range ratios. The Fib-Gen Repaired group showed strong trends $(p=0.07$ and $p=0.06$ ) of improved biomechanical behaviours relative to the Injured group, yet torsional stiffness and torque range ratios remained significantly lower than the Intact condition. $\mathrm{a}=p<0.05, \mathrm{~b}=p<0.01$ compared to the corresponding Intact condition within the same specimen.

the stiffness ratio in the Repaired group when compared to the Injured group ( $p=0.09$, Fig. $6 \mathrm{c})$. The axial range of motion ratio was larger for the Injured group than for the repaired group, although no significant difference was detected between the two groups (Table 1).

A typical torque-rotation curve from the cyclic torsional test of an injured specimen is shown in Fig. 6d. Results indicated that the torsional stiffness and torque range ratios were greatly decreased with injury ( $p<0.001$, Fig. 6e,f). There was a trend of restoration in these parameters for the Repaired group relative to the Injured as shown by increases in the torsional stiffness and torque range ratio ( $p=0.07$ and 0.09, respectively, Fig. 6e,f).
From the stress relaxation test, the ratio of the curvefitted relaxation time constant was similar for the Injured and Repaired groups, with no significant differences detected between the two groups (Table 1).

\section{Subcutaneous implantation}

The degradation of Fib-Gen discs following subcutaneous implantation into rats appeared to be partial after 8 weeks and extensive to nearly complete after 16 weeks (Fig. 7). At 8 weeks, Fib-Gen disc diameter diminished by $40.5 \pm 2.9 \%$ to $2.97 \pm 0.15 \mathrm{~mm}$ ( $n=3$ gels). At 16 weeks, small pieces of Fib-Gen were observable, however, the gel dimension was not measurable due to extensive degradation (Fig. 7d- 

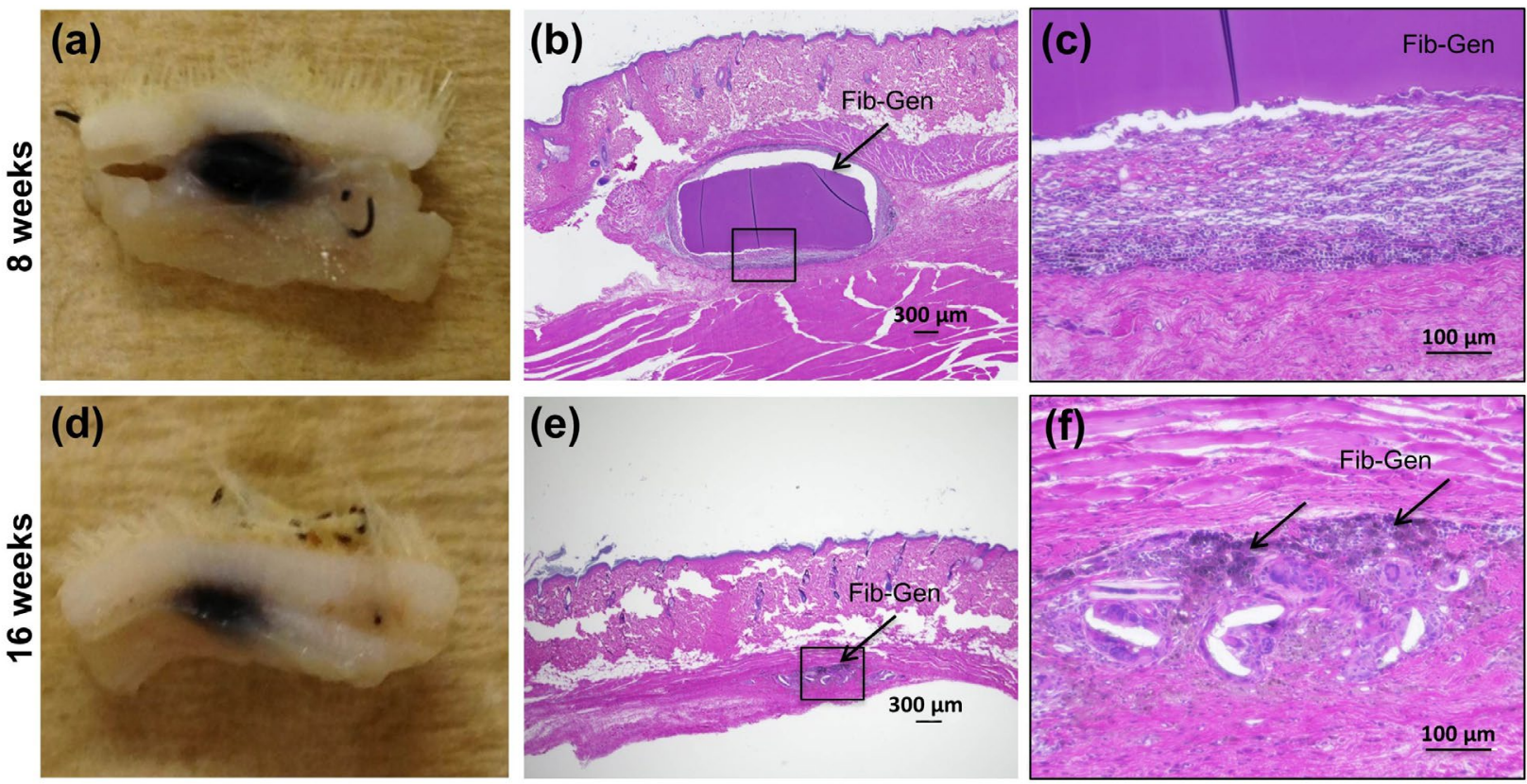

Fig. 7. In vivo degradation of Fib-Gen and biocompatibility of Fib-Gen. Subcutaneous implants harvested after 8 weeks $(\mathbf{a}, \mathbf{b}, \mathbf{c})$ and 16 weeks $(\mathbf{d}, \mathbf{e}, \mathbf{f})$. H\&E staining of implant sections at $4 x(\mathbf{b}, \mathbf{e})$ and 20x $(\mathbf{c}, \mathbf{f})$ showed partial degradation and cell infiltration at 8 weeks $(\mathbf{b}, \mathbf{c})$ and nearly complete resorption at 16 weeks (e,f). Moderate inflammatory response was observed based on qualitative assessment of the immune cells and fibrous tissues (c,f).

f). H\&E staining of Fib-Gen implants, when qualitatively compared to previous studies on inflammatory response to subcutanously implanted biomaterials in rats, showed the presence of immune cells at both time points (Fig. $7 \mathrm{~b}, \mathrm{c}, \mathrm{e}, \mathrm{f})$ with the characteristic of fibrous tissue apparent at 8 weeks (Fig. 7b,c). At 16 weeks, the small remnant area of Fib-Gen showed full infiltration of remaining immune cells and indicated nearly complete resorption (Fig. 7e,f).

\section{Discussion}

There is an unmet clinical need for an adhesive biomaterial capable of repairing small and large AF defects resulting from degeneration, needle injection, and herniation. Such a biomaterial may also be useful to augment and improve current discectomy procedures by sealing the AF following tissue removal, which may prevent re-herniation and slow progressive IVD degeneration. Fib-Gen is an adhesive hydrogel that is cytocompatible and previously formulated to biomechanically match shear mechanical properties of native AF. In this study, Fib-Gen hydrogel was used to repair a large box-cut AF defect in a bovine caudal IVD model using organ culture and biomechanical testing, and was assessed for its ability to achieve our AF repair design requirements of being strongly adhesive, biocompatible, able to withstand repetitive loading, and injectable for easy delivery at the time of surgery. The in vivo degradation rate and biocompatibility of Fib-Gen was also assessed with subcutaneous implantation in rats.

Fib-Gen repair successfully prevented IVD height loss, and inhibited NO expression while remaining fully integrated with the native AF tissue in organ culture. FibGen gel interpenetrated with the damaged AF collagen structure, remained well adhered to the native AF tissues following 14,000 cycles of simulated physiological loading, and maintained in a stable volume filling the defect space. Results therefore suggested that the defect remained effectively sealed by Fib-Gen throughout this 6-day organ culture experiment. We infer that the addition of the genipin cross-linker in our Fib-Gen formulation increased the gel stiffness sufficiently to maintain continuity of strain transfer between the gel and the surrounding AF at the injured site thereby retaining NP pressurisation, GAG content, and IVD height.

Previously, Fib-Gen exhibited cytocompatibility by allowing growth and differentiation of human AF cells when plated on solidified gels in culture (Guterl et al., 2014; Schek et al., 2011). The current study further demonstrated that native AF cells within cultured bovine IVDs retained high cell viability near the repair site. In addition, Fib-Gen was shown to be cytocompatible with NP cells, as indicated by comparably high NP cell viability in repaired IVDs. Importantly, native IVD cells were also found to migrate into the Fib-Gen gel even in this short term 6-day experiment as demonstrated on cryosections (Fig. 5a; DAPI staining shows cell migration into the Fib-Gen gel although dark specks prevented validation of cell viability) and with confocal imaging (Fig. 5b, cyan staining of calcein/DAPI demonstrated live cells migrated into the depth of Fib-Gen). We anticipate that IVD cells could populate the Fib-Gen gel in vivo, and therefore it is suggested that Fib-Gen may allow tissue regeneration in the repair site over a longer duration. These compelling results motivate a comprehensive evaluation of long-term AF repair outcomes in an in vivo model.

The reduction of nitric oxide release into the media in the repaired IVDs compared to Injured group indicated that the Fib-Gen sealant allowed native IVD cells to adapt to their cellular environment and may have prevented an 
inflammatory response. However, direct measurement of anti-inflammatory capacity of Fib-Gen requires future investigations. This finding, in addition to the high cell viability of AF cells immediately surrounding the repair site, suggests that Fib-Gen is likely to have a good safety profile if used as an AF repair biomaterial.

An effective AF repair material must be able to resist large and immediate physiological loads and deformation observed in human spines (Wilke et al., 2003). Biomechanical testing showed that Fib-Gen was able to fully restore the compressive stiffness of repaired motion segments to intact levels, is consistent with observed functional restoration (IVD height loss prevention) in organ culture. The compressive stiffness of the Injured group was counter-intuitively increased when compared to the intact state of the same specimen. This stiffening effect may be associated with compaction and the nonlinear behaviour of IVD under compression and was observed in the nonsignificant trend of larger values for axial range of motion in Injured samples. This behaviour may be considered as an early pathological change in the load support characteristic, where the axial load is distributed into the AF region. The tensile stiffness had a trend of improvement by Fib-Gen repair compared to the Injured group, suggesting that the stiffness of Fib-Gen was sufficient to provide partial restoration of the integrity of the AF structure.

Torsional properties of IVD were the biomechanical parameters most sensitive to AF injury, as demonstrated by the lowest ratio of the torsional stiffness and torque range for the injured condition relative to intact conditions, and this finding is consistent with prior results (Michalek and Iatridis, 2012). Repair with Fib-Gen showed a trend of partial restoration of both torsional stiffness and torque range compared to the Injured group, which also indicated partial restoration of the AF integrity. However, the Repaired group had torsional properties significantly lower than the intact condition, which was likely attributed to cracks observed in the mid region of the bulk portion of the gel (data not shown) that might have been developed due to tensile strains induced during torsional testing at angles of $\pm 3^{\circ}$. This is further supported by the finding that stress relaxation tests performed after the torsional test showed no difference in the stress relaxation time constant between Injured and Repaired groups, suggesting a lack of NP pressurisation due to Fib-Gen cracking. Nevertheless, no herniation of Fib-Gen was observed throughout the test in all repaired specimens.

A large AF injury was used in this study to simulate a defect that might be created following a microdiscectomy procedure. The box-cut defect included the sharp corners present from the cut wound of discectomy but does not perfectly replicate the rough edges and distorted shape of AF defect following herniation. The simulated defect limited the amount of NP removal to approximately $15 \%$ of total NP volume, putting Fib-Gen to the additional challenge of simulating an ideal AF repair strategy that could restore AF integrity and retain NP pressurisation. Results demonstrated full restoration of compressive stiffness and partial restoration of tensile and torsional stiffness suggesting overall improvement of NP pressurisation and AF integrity (Michalek and
Iatridis, 2012) following a large defect injury. It should be noted that the biomechanical testing regime performed in this study represented a first evaluation of Fib-Gen's performance in situ, aiming to determine if the gel can restore or improve IVD biomechanics under physiological load. To determine whether Fib-Gen can prevent reherniation in a large defect, a test under more extreme loading conditions designed to induce herniation is required, e.g., simultaneous compression and bending at higher magnitudes. Nonetheless, we expect Fib-Gen would perform even better in small AF injury conditions, but this was not evaluated because the altered biomechanics and biology from small defects in a short timeframe are difficult to detect due to the biological and experimental variations.

Because AF repair biomaterials have to be able to immediately withstand repetitive spinal loading, such biomaterials should have an optimum degradation rate that promotes concurrent and sufficient tissue regeneration in order to provide continous biomechanical restoration. While the fibrin sealant is widely used for general tissue repair, it is known to degrade rapidly and have insufficient biomechanical strength, suggesting it may not be suitable as AF repair biomaterial (Dickneite et al., 1994; Dickneite et al., 2003; Lorentz et al., 2011). Previously, Dare and coworkers (Dare et al., 2009) have developed genipin crosslinked fibrin hydrogel for cartilage tissue engineering, and reported that fibrin gel was extensively degraded after 3 weeks when subcutaneously implanted in rats, but that genipin enhanced the stability of fibrin gel with limited degradation at 3 weeks. In this study, we found that our FibGen implants degraded relatively slowly with $40 \%$ change in dimension at 8 weeks and a nearly complete resorption occurring after 16 weeks. Fib-Gen was therefore found to have greatly enhanced longevity in vivo as compared to fibrin data found in the literature. The slow degradation rate of Fib-Gen may serve as an advantage, allowing AF to be sealed to retain NP pressurisation for an extended period of time in order to enable tissue regeneration, which occurs slowly in the IVDs and more slowly under the inflamed environment of injury and degeneration. The current results show promise for an AF repair strategy with a single Fib-Gen sealant injection that can provide biomechanical restoration and allow tissue regeneration over relatively long periods. Fib-Gen can also be further modified to include matrix proteins to promote better cell adhesion (Guterl et al., 2014) or can be used in combination with other biomaterials to promote more rapid cell infiltration and further accelerate tissue regeneration.

Following subcutaneous implantation in the rats, a moderate inflammatory response to Fib-Gen was observed by the presence of immune cells and fibrous tissue. This response was not surprising as such reaction is expected to be induced by thrombin and the xenogeneic bovine fibrinogen that were used to prepare the Fib-Gen in this study. Similar xenogeneic reactions to human fibrin materials were observed to a greater extent in rat subcutaneous tissue in a previous study (Dare et al., 2009), and the addition of genipin was reported to reduce the immune response. In comparison to such study, our results implied that the presence of genipin in our Fib-Gen formulation is not likely to have a negative effect on the 
gel biocompatibility and the observed immune response. Translational application of Fib-Gen for pre-clinical testing or human clinical trials may involve re-formulation of our Fib-Gen using species matched fibrinogen and thrombin to reduce immune response to fibrin material, and this is expected to further enhance Fib-Gen stability and biocompatibility.

In conclusion, this study evaluated the performance of Fib-Gen to repair large AF defects utilising organ culture and biomechanical testing procedures on bovine caudal motion segments, as well as assessed the in vivo degradation rate and biocompatibility in rats, in protocols designed to robustly assess the biomaterial and minimise large animal usage. A single injection of FibGen adhesive hydrogel withstood repetitive compressive loading, provided functional biological and biomechanical restoration in large animal organ culture, suggesting it may have a role as a biocompatible, bio-absorbable adhesive biomaterial for regenerative repair of IVD with ruptured AF. Fib-Gen restored IVD height and compressive properties and partially restored other biomechanical behaviours of IVD motion segments under a range of physiological load without herniation, demonstrating that Fib-Gen has potential to repair large AF defects and may reduce the re-herniation rate following lumbar discectomy procedures. Cracks observed following loading conditions with high tensile strains suggested that some formula enhancements are warranted. However, Fib-Gen was able to improve biomechanical function and is likely to be superior to fibrin alone as an AF repair material because of its increased mechanical stiffness, slower degradation rates, and comparable in vivo biocompatibility. Overall, injectable Fib-Gen shows promise for use in AF repair strategies that may provide significant structural stability necessary to prevent or slow the degeneration of injured IVDs.

\section{Acknowledgement}

Funded by NIAMS/NIH grant R01 AR057397, a consortium grant from AO Exploratory Research Board, and Studienstiftung des deutschen Volkes award. We also gratefully acknowledge the support of Dr. Devina Purmessur, Mr. Damien Laudier, and Mr. Philip Nasser for their helpful assistance on several technical aspects of this project. We wish to confirm that there are no known conflicts of interest associated with this publication and there has been no significant financial support for this work that could have influenced its outcome.

\section{References}

Adams MA, Dolan P (2012) Intervertebral disc degeneration: evidence for two distinct phenotypes. J Anat 221: 497-506.

Ahlgren BD, Lui W, Herkowitz HN, Panjabi MM, Guiboux JP (2000) Effect of anular repair on the healing strength of the intervertebral disc: a sheep model. Spine 25: 2165-2170.
Bron JL, van der Veen AJ, Helder MN, van Royen BJ, Smit TH (2010) Biomechanical and in vivo evaluation of experimental closure devices of the annulus fibrosus designed for a goat nucleus replacement model. Eur Spine J 19: 1347-1355.

Buser Z, Kuelling F, Liu J, Liebenberg E, Thorne KJ, Coughlin D, Lotz JC (2011) Biological and biomechanical effects of fibrin injection into porcine intervertebral discs. Spine 36: E1201-1209.

Carragee EJ, Don AS, Hurwitz EL, Cuellar JM, Carrino JA, Herzog R (2009) Does discography cause accelerated progression of degeneration changes in the lumbar disc: a ten-year matched cohort study. Spine 34: 2338-2345.

Chan SC, Ferguson SJ, Wuertz K, Gantenbein-Ritter B (2011) Biological response of the intervertebral disc to repetitive short-term cyclic torsion. Spine 36: 2021-2030.

Chan SC, Gantenbein-Ritter B (2012a) Intervertebral disc regeneration or repair with biomaterials and stem cell therapy - feasible or fiction? Swiss Med Wkly 142: w13598.

Chan SC, Gantenbein-Ritter B (2012b) Preparation of intact bovine tail intervertebral discs for organ culture. $\mathrm{J}$ Vis Exp 60: 3490.

Dare EV, Griffith M, Poitras P, Kaupp JA, Waldman SD, Carlsson DJ, Dervin G, Mayoux C, Hincke MT (2009) Genipin cross-linked fibrin hydrogels for in vitro human articular cartilage tissue-engineered regeneration. Cells Tissues Organs 190: 313-325.

Demers CN, Antoniou J, Mwale F (2004) Value and limitations of using the bovine tail as a model for the human lumbar spine. Spine 29: 2793-2799.

DePalma MJ, Ketchum JM, Saullo TR, Laplante BL (2012) Is the history of a surgical discectomy related to the source of chronic low back pain? Pain Physician 15: E53-E58.

Deyo RA, Weinstein JN (2001) Low back pain. N Engl J Med 344: 363-370.

Dickneite G, Czech J, Keuper H (1994) Formation of fibrin monomers in experimental disseminated intravascular coagulation and its inhibition by recombinant hirudin. Circ Shock 42: 183-189.

Dickneite G, Metzner H, Pfeifer T, Kroez M, Witzke G (2003) A comparison of fibrin sealants in relation to their in vitro and in vivo properties. Thromb Res 112: 73-82.

Elliott DM, Yerramalli CS, Beckstein JC, Boxberger JI, Johannessen W, Vresilovic EJ (2008) The effect of relative needle diameter in puncture and sham injection animal models of degeneration. Spine 33: 588-596.

Freemont AJ, Peacock TE, Goupille P, Hoyland JA, O'Brien J, Jayson MI (1997) Nerve ingrowth into diseased intervertebral disc in chronic back pain. Lancet 350: 178181.

Gantenbein B, Grunhagen T, Lee CR, van Donkelaar CC, Alini M, Ito K (2006) An in vitro organ culturing system for intervertebral disc explants with vertebral endplates: a feasibility study with ovine caudal discs. Spine 31: 2665-2673.

Guterl CC, See EY, Blanquer SB, Pandit A, Ferguson SJ, Benneker LM, Grijpma DW, Sakai D, Eglin D, Alini M, Iatridis JC, Grad S (2013) Challenges and strategies 
in the repair of ruptured annulus fibrosus. Eur Cell Mater 25: $1-21$.

Guterl CC, Torre OM, Purmessur D, Dave K, Likhitpanichkul M, Hecht A, Nicoll SP, Iatridis JC (2014) Characterization of mechanics and cytocompatibility of fibrin-genipin annulus fibrosus sealant with the addition of cell adhesion molecules. Tissue Eng Part A Epub ahead of print PMID 24683414.

Haglund L, Moir J, Beckman L, Mulligan KR, Jim B, Ouellet JA, Roughley P, Steffen T (2011) Development of a bioreactor for axially loaded intervertebral disc organ culture. Tissue Eng Part C Methods 17: 1011-1019.

Holm S, Maroudas A, Urban JP, Selstam G, Nachemson A (1981) Nutrition of the intervertebral disc: solute transport and metabolism. Connect Tissue Res 8: 101-119.

Iatridis JC, Hecht AC (2012) Commentary: Does needle injection cause disc degeneration? News in the continuing debate regarding pathophysiology associated with intradiscal injections. Spine J 12: 336-338.

Iatridis JC, Nicoll SB, Michalek AJ, Walter BA, Gupta MS (2013) Role of biomechanics in intervertebral disc degeneration and regenerative therapies: what needs repairing in the disc and what are promising biomaterials for its repair? Spine J 13: 243-262.

Illien-Junger S, Pattappa G, Peroglio M, Benneker LM, Stoddart MJ, Sakai D, Mochida J, Grad S, Alini M (2012) Homing of mesenchymal stem cells in induced degenerative intervertebral discs in a whole organ culture system. Spine 37: 1865-1873.

Intaglietta M, Johnson PC, Winslow RM (1996) Microvascular and tissue oxygen distribution. Cardiovasc Res 32: 632-643.

Jacobs JJ, Andersson GB, Bell J-E, Weinstein SL, Dormans JP, Furman MB, Lane N, Puzas, JE, St. Clair EW, Yelin EH (2011) Spine: low back and neck pain. In: United States Bone and Joint Initiative: The Burden of Musculoskeletal Diseases in the United States, 2nd ed. American Academy of Orthopaedic Surgeons, Rosemont, IL, chapter 2, pp 21-25.

Johannessen W, Vresilovic EJ, Wright AC, Elliott DM (2004) Intervertebral disc mechanics are restored following cyclic loading and unloaded recovery. Ann Biomed Eng 32: 70-76.

Johannessen W, Cloyd JM, O'Connell GD, Vresilovic EJ, Elliott DM (2006) Trans-endplate nucleotomy increases deformation and creep response in axial loading. Ann Biomed Eng 34: 687-696.

Junger S, Gantenbein-Ritter B, Lezuo P, Alini M, Ferguson SJ, Ito K (2009) Effect of limited nutrition on in situ intervertebral disc cells under simulated-physiological loading. Spine 34: 1264-1271.

Katz JN (2006) Lumbar disc disorders and low-back pain: socioeconomic factors and consequences. J Bone Joint Surg Am 88 Suppl 2: 21-24.

Korecki CL, MacLean JJ, Iatridis JC (2007) Characterization of an in vitro intervertebral disc organ culture system. Eur Spine J 16: 1029-1037.

Korecki CL, Costi JJ, Iatridis JC (2008a) Needle puncture injury affects intervertebral disc mechanics and biology in an organ culture model. Spine 33: 235-241.
Korecki CL, MacLean JJ, Iatridis JC (2008b) Dynamic compression effects on intervertebral disc mechanics and biology. Spine 33: 1403-1409.

Lee CR, Iatridis JC, Poveda L, Alini M (2006) In vitro organ culture of the bovine intervertebral disc: effects of vertebral endplate and potential for mechanobiology studies. Spine 31: 515-522.

Leung VY, Chan WC, Hung SC, Cheung KM, Chan D (2009) Matrix remodeling during intervertebral disc growth and degeneration detected by multichromatic FAST staining. J Histochem Cytochem 57: 249-256.

Li Z, Liu H, Yang H, Wang J, Wang H, Zhang K, Ding W, Zheng Z (2014) Both expression of cytokines and posterior annulus fibrosus rupture are essential for pain behavior changes induced by degenerative intervertebral disc: An experimental study in rats. J Orthop Res 32: 262272.

Lorentz KM, Kontos S, Frey P, Hubbell JA (2011) Engineered aprotinin for improved stability of fibrin biomaterials. Biomaterials 32: 430-438.

Malhotra NR, Han WM, Beckstein J, Cloyd J, Chen W, Elliott DM (2012) An injectable nucleus pulposus implant restores compressive range of motion in the ovine disc. Spine 37: E1099-1105.

Malonzo C, Chan SC, Kabiri A, Eglin D, Grad S, Bonel HM, Benneker LM, Gantenbein-Ritter B (2013) A papain-induced disc degeneration model for the assessment of thermo-reversible hydrogel-cells therapeutic approach. J Tissue Eng Regen Med Epub ahead of print PMID 23303720.

Masuda K, Aota Y, Muehleman C, Imai Y, Okuma M, Thonar EJ, Andersson GB, An HS (2005) A novel rabbit model of mild, reproducible disc degeneration by an anulus needle puncture: correlation between the degree of disc injury and radiological and histological appearances of disc degeneration. Spine 30: 5-14.

Michalek AJ, Funabashi KL, Iatridis JC (2010) Needle puncture injury of the rat intervertebral disc affects torsional and compressive biomechanics differently. Eur Spine J 19: 2110-2116.

Michalek AJ, Iatridis JC (2012) Height and torsional stiffness are most sensitive to annular injury in large animal intervertebral discs. Spine J : 12: 425-432.

Oshima H, Ishihara H, Urban JP, Tsuji H (1993) The use of coccygeal discs to study intervertebral disc metabolism. J Orthop Res 11: 332-338.

Ohshima H, Urban JP, Bergel DH (1995) Effect of static load on matrix synthesis rates in the intervertebral disc measured in vitro by a new perfusion technique. J Orthop Res 13: 22-29.

Osti OL, Vernon-Roberts B, Moore R, Fraser RD (1992) Annular tears and disc degeneration in the lumbar spine. A post-mortem study of 135 discs. J Bone Joint Surg Br 74: 678-682.

Paul CP, Zuiderbaan HA, Zandieh Doulabi B, van der Veen AJ, van de Ven PM, Smit TH, Helder MN, van Royen BJ, Mullender MG (2012) Simulated-physiological loading conditions preserve biological and mechanical properties of caprine lumbar intervertebral discs in ex vivo culture. PloS one 7: e33147. 
Pearcy MJ, Tibrewal SB (1984) Axial rotation and lateral bending in the normal lumbar spine measured by three-dimensional radiography. Spine 9: 582-587.

Purmessur D, Freemont AJ, Hoyland JA (2008) Expression and regulation of neurotrophins in the nondegenerate and degenerate human intervertebral disc. Arthritis Res Ther 10: R99.

Sato K, Kikuchi S, Yonezawa T (1999) In vivo intradiscal pressure measurement in healthy individuals and in patients with ongoing back problems. Spine 24: 2468-2474.

Schek RM, Michalek AJ, Iatridis JC (2011) Genipincrosslinked fibrin hydrogels as a potential adhesive to augment intervertebral disc annulus repair. Eur Cell Mater 21: 373-383.

Schneider CA, Rasband WS, Eliceiri KW (2012) NIH Image to ImageJ: 25 years of image analysis. Nat Methods 9: 671-675.

Showalter BL, Beckstein JC, Martin JT, Beattie EE, Espinoza Orias AA, Schaer TP, Vresilovic EJ, Elliott DM (2012) Comparison of animal discs used in disc research to human lumbar disc: torsion mechanics and collagen content. Spine 37: E900-907.

Sobajima S, Kompel JF, Kim JS, Wallach CJ, Robertson DD, Vogt MT, Kang JD, Gilbertson LG (2005) A slowly progressive and reproducible animal model of intervertebral disc degeneration characterized by MRI, X-ray, and histology. Spine 30: 15-24.

Steffen T, Rubin RK, Baramki HG, Antoniou J, Marchesi D, Aebi M (1997) A new technique for measuring lumbar segmental motion in vivo. Method, accuracy, and preliminary results. Spine 22: 156-166.

Sung HW, Huang RN, Huang LL, Tsai CC (1999) In vitro evaluation of cytotoxicity of a naturally occurring cross-linking reagent for biological tissue fixation. J Biomater Sci Polym Ed 10: 63-78.

Vernon-Roberts B, Fazzalari NL, Manthey BA (1997) Pathogenesis of tears of the anulus investigated by multiple-level transaxial analysis of the T12-L1 disc. Spine 22: 2641-2646.

Walter BA, Korecki CL, Purmessur D, Roughley PJ, Michalek AJ, Iatridis JC (2011) Complex loading affects intervertebral disc mechanics and biology. Osteoarthritis Cartilage 19: 1011-1018.

Walter BA, Illien-Junger S, Nasser PR, Hecht AC, Iatridis JC (2014) Development and validation of a bioreactor system for dynamic loading and mechanical characterization of whole human intervertebral discs in organ culture. J Biomech 47: 2095-2101

Wilke HJ, Neef P, Caimi M, Hoogland T, Claes LE (1999) New in vivo measurements of pressures in the intervertebral disc in daily life. Spine 24: 755-762.

Wilke HJ, Rohlmann A, Neller S, Graichen F, Claes L, Bergmann G (2003) A novel approach to determine trunk muscle forces during flexion and extension: a comparison of data from an in vitro experiment and in vivo measurements. Spine 28: 2585-2593.

Wiseman MA, Birch HL, Akmal M, Goodship AE (2005) Segmental variation in the in vitro cell metabolism of nucleus pulposus cells isolated from a series of bovine caudal intervertebral discs. Spine 30: 505-511.

\section{Discussion with Reviewers}

Reviewer I: Do the authors conclude that the even lower decrease of disc height in the Repair model compared to the intact disc presented in Fig. 4 is the result of the significant increase of compressive stiffness in the Repair group relative to the intact group presented in Table 1? In other words, does the genipin cross-linking of the fibrin gel make the repair group stiffer than the intact disc?

Authors: While the mean of the \% disc height loss in the Repaired group appeared to be lower than that of the Intact group (Fig. 4), the variation was large. We therefore interpreted this result as 'no significant difference' between the Repaired and Intact groups. Biomechanical testing further indicated that the compressive stiffness of the Repaired group (Table 1, Fig. 6) was also nearly identical to the intact value before the repair (i.e., ratio $=1.02$ ), further suggesting no change in compressive stiffness in the repair group relative to the intact condition. In fact, the Injured group (without repair) demonstrated an increase in compressive stiffness as compared to the intact value providing evidence that the Fib-Gen repair prevented some of the stiffening observed in the Injured group.

Reviewer II: The genipin crosslinking served to provide mechanical strength but also inhibited cell infiltration for very longer periods of time ( $>8$ weeks) and thus inhibited gel remodelling. Should this be considered a benefit of the gel as bio-inert, or a weakness of the FibGen formulation? Authors: Our current formulation is generally 'bio-inert' and our results suggested that the cell infiltration was not inhibited but occurred slowly at the gel interface and progressed over time into the gel. At 8 weeks following subcutaneous implantation, we found that the gel diameter was decreased by approximately $40 \%$, suggesting partial cell infiltration and absorption of the gel had occurred. The gel's extended longevity of Fib-Gen is an advantage because it is important for the AF to remain sealed with the gel to retain NP pressurisation during the repair process, which occurs very slowly in the IVD and more slowly under the inflamed environment of injury and degeneration. We believe that the current Fib-Gen formulation shows promise for an AF repair strategy as an injectable adhesive hydrogel that provides extended biomechanical restoration and allows tissue regeneration in the long term. Fib-Gen can also be further modified to include matrix proteins to promote cell adhesion (Guterl et al, 2014) or can be used in combination with other biomaterials to promote more rapid cell infiltration and further accelerate tissue regeneration.

Reviewer III: The use of hypoxic conditions for the organ culture is interesting; however, I am not sure that the organ itself can be considered to exist in a hypoxic environment. It is true that the individual disc cells are subjected to a low oxygen environment due to steep gradients within the disc, but I wonder if limiting the oxygen concentration at the boundaries of the disc is perhaps too demanding. This would be an interesting discussion point, as the "proper" conditions for organ culture are still being defined.

Authors: The pathway for oxygen supply to the IVD includes capillaries at the subchondral plate that penetrate 
into the cartilaginous endplate, and at the AF periphery (Urban et al., 1977). The oxygen tension in vivo reduces from approximately $13 \%$ (100 mm $\mathrm{Hg}$ partial pressure) in the arteries to approximately $4 \%(25-30 \mathrm{~mm} \mathrm{Hg})$ in the capillaries, while the value in general tissues and in the veins is typically approximated to be $5 \%$ or less $(<40 \mathrm{~mm}$ $\mathrm{Hg}$ ) (Intaglietta et al., 1996). The oxygen tension within the IVD was previously measured to be approximately $5 \%$ $(5 \mathrm{kPa})$ near the edge of the outer AF, and approximately $7 \%(7 \mathrm{kPa})$ near the endplate (Holm et al., 1981). Therefore, we believe that the use of hypoxic condition in the organ culture more closely simulate the physiological level of oxygen tension at the IVD boundaries. An optimum oxygen tension is yet to be refined, but we believe that the currently used level of $5 \%$ or slightly higher is reasonable.

\section{Additional Reference}

Urban J, Holm S, Maroudas A, Nachemson A (1977) Nutrition of the intervertebral disk. An in vivo study of solute transport. Clin Orthop Relat Res 129: 101-114. 